\title{
Current Work Practice and Users' Perspectives on Visualization and Interactivity in Business Intelligence
}

\author{
Wolfgang Aigner \\ Institute of Software Technology \& Interactive Systems \\ Vienna University of Technology \\ Vienna, Austria \\ aigner@ifs.tuwien.ac.at
}

\begin{abstract}
Business Intelligence (BI) provides methods for analyzing business-critical information and supports decisionmaking processes. Interactivity or the possibility to engage in an active discourse with the representation lies at the core of Information Visualization and Visual BI. However, current work practice and users' perspectives on visualization and interactivity in BI have not been investigated yet. As a first step in this direction, a qualitative empirical study was conducted among six IT-managers working in the field of BI. Main results are that interactive visual methods in BI are not used very often in practice. One of the main reasons reported is the fact that visualization is still two steps ahead and at the moment mostly more basic problems concerning data gathering, data modeling and data quality prevail. Moreover, most users are used to work with numbers and tables and are not aware of the possibilities in terms of visualization. However, the interviewed IT-managers acknowledged that using more interactive visual methods in BI would be beneficial for users.
\end{abstract}

Keywords-visual business intelligence; information visualization; interactivity; qualitative empirical study.

\section{INTRODUCTION}

The development and the success of many organizations to a large extent depend on how well and how timely they manage to gain insight into collected data. For example, consider the case of a simple shoe store. Gathering valuable information from collecting and analyzing data regarding customer frequency, seasonal demand variations, price politics of competitors, anticipated hypes, etc., helps the manager in making better informed business decisions. Personnel planning could be improved, warehousing costs lowered and competitors might be outperformed by basing decisions upon collected data and hard facts rather than guessing and gut feeling. Business Intelligence (BI) provides methods for analyzing business-critical information (e.g., about customers, competitors, economic environment, internal processes) to make high quality decisions and view the business's strengths and weaknesses on a daily basis. During the last decade, the possibilities to both generate and collect data and information have grown tremendously. Traditional manual methods of data analysis such as spreadsheets, ad-hoc queries or simple diagrams cannot cope with this amount of data and information. We need new methods and tools that can intelligently and (semi-)automatically transform data into information and, furthermore, synthesize knowledge. Considering these technological developments, the importance of BI is growing [1].

Information Visualization (InfoVis) is an important asset in this set of tools. By utilizing the powerful human perceptual system that is extremely efficient in processing visual input, visualization can help to make sense of data, explore complex information spaces, or spot patterns and relationships within the data. Visual Business Intelligence is a term coined by Few [2] and describes the application of InfoVis in the domain of BI to represent and analyze business data visually. User interaction is one of the most important elements in visualization or even the core [3]. As Saraiya et al. found out in a study [4], users prefer inferior visualizations with interaction over superior visualizations without interaction features. Further, they mention that visual representations provide only an initial direction to the data and their meaning, while through the combination of visual representations and appropriate interaction mechanisms, the users achieve insights into the data.

However, there are almost no systematic accounts for investigating the users' perspective on the value and role of interactivity in visualization and data analysis [5], [6]. More generally, in a recent work on empirical evaluation in InfoVis [7], the authors identify 'understanding environments and work practices' as one of seven basic evaluation scenarios. This type of evaluation is reported to be highly underrepresented and the authors discuss encouraging more evaluations to be conducted and published. The aim of this paper is to add to that by assessing the role as well as the benefits and limitations of interactivity and visual methods for business data analysis as perceived by users.

\section{RELATED WORK}

$\mathrm{BI}$ is a complex and multifaceted area with a history of more than 40 years [8]. BI methods and techniques are intended to support managers and experts in business processes from operations to strategic decisions. BI, and more specifically business analytics, has recently received growing interest as a vital asset for businesses today [9]. 
One of the key factors that impacts business is the ability to gain relevant information from the growing amounts of data in order to make better decisions. To achieve this, several experts in the field call for more interactive navigation and exploration functionalities along with advanced visualization possibilities [10], [11]. The current popular reports and dashboards represent only a fraction of Visual BI that supports mainly presentation tasks (classical reporting). More sophisticated and interactive techniques need to be utilized to provide support for analysis and exploration. A review of current BI software products [12] found that currently used visualizations are often ineffective in communicating important information. Related to that Kohlhammer et al. [13] report that broadly used BI products did not improve interactivity during the last years, even to the contrary, the moves towards web-based versions even worsened the situation. Along with the rise of business analytics, Visual BI has grown very fast lately and can be traced by recent mergers and acquisitions where large BI vendors have acquired companies specialized in visual data analysis [14].

Regarding interactivity in general, only recently a small amount of studies has focused on the value of interactivity. A study [15] on an interactive learning environment with varying degrees of interactivity shows that there is a "greater preference for the interactive session and prevailing belief that interactivity is better than none" [15]. However, the study does not indicate that better learning had been achieved when interactivity was involved. The author concluded that "basic knowledge about a domain may be best gained passively, but that knowledge about how to behave and what questions to ask in that domain are best gained through active involvement" [15]. In a study conducted by Saraiya et al. [4] users preferred inferior visualizations with interaction over superior static visualizations. Overall, interactivity is considered to be a vital element in InfoVis but models and empirical evidence are scarce in literature [5], [6].

\section{METHOD}

From a methodological point of view, this work is based on qualitative semi-structured interviews. This allowed for getting an overview of the interviewee's main foci without being too constraining. The questions asked and issues discussed in semi-structured interviews were informed by cognitive and post-cognitive models and theories. Specifically, the analysis goals, use-cases, constraints, frequency of use in general, as well as the perceived role and value of interactivity in particular, have been investigated.

\section{A. Research Question and Hypotheses}

The main research question to be answered in the study was: What is the perceived value and role of interactivity in visual methods for business data analysis? Based on that, a set of three hypotheses was derived:
H1. Most visual methods currently applied in Business Intelligence are static or employ only very limited forms of interactivity.

$\mathrm{H} 2$. Increasing the interactivity of visual methods is desired by users.

H3. According to users, interactivity helps to gain information and knowledge in business data analysis.

To answer the research question and shed light on the hypotheses, the following areas have been investigated:

- Which visual methods are currently applied and what are they used for?

- Are these methods paper or computer-based and are they static or interactive?

- How are the visual methods used?

- How important are visual methods for business data analysis and for decision-making?

- What does interactivity mean to the person interviewed?

- What value is ascribed to interactivity?

\section{B. Interview Partners}

Six IT managers of Austrian businesses, who are responsible for introducing and maintaining the BI infrastructure in their company, were asked to present and explain visual methods that they use. This group of persons has been selected because they know the BI tools in use very well and have knowledge about their respective users. Moreover, these persons are presumably more knowledgeable in estimating the possible value or impact of introducing more interactivity in their BI landscape. The industry sectors have not been restricted; quite on the contrary, a more heterogeneous set of sectors is considered beneficial in order to retrieve a broader view of general BI system usage. The interview partners were selected by contacting a number of IT managers who fit into the characteristics described above via email, using a one-page info sheet that described the planned study.

All six interviewed IT-managers are male and between 30 and 39 years old (5 persons), with one interview partner being 55-59 of age. Most of the interview partners (IPs) are at the level of team/group leaders (4 persons). One IP is an IT expert and one IP is a division manager. The industrial sectors within which IPs work are relatively heterogeneous: health, media, production, mechanical engineering, and financial market (2). All IPs have more than four years of experience in the field of BI, with the majority having about 10 years of experience.

\section{Materials \& Procedure}

An interview guideline was prepared that consisted of four parts (introduction, visual methods, interactivity in visual methods, and demographic data). The interview length ranged between 30 and 45 min., with one interview taking considerably longer (74 min.). The interviews were conducted either at the workplace of the interview partners (4), the office of the author (1), or via telephone (1). The 
companies of four interview partners are located in Vienna, one in Salzburg, and one in Tyrol. All interviews were audiotaped and held in form of an oral dialogue. Generally, no examples or computer demos were used, neither by the interviewer (I) nor by the interview partners (IP). Only one interview partner demonstrated the running system of the respective enterprise to the interviewer.

\section{Analysis approach}

The interview transcripts were analyzed qualitatively using an open coding scheme. A data-driven coding approach was pursued that was derived directly from the data via qualitative interpretation by the author [16]. The coding scheme was derived incrementally by first identifying and annotating themes in logically connected chunks of text by the author. In a second step, the identified low-level themes were grouped and organized hierarchically based on topic and similarity in order to structure the identified themes.

\section{RESUlts}

The following summary is structured along the three main themes BI, visualization, and interactivity, which also determined the main structure of the interviews. As the interviews were held in German, all presented quotes have been translated to English by the author.

\section{A. Business Intelligence}

As an introductory question, the interview partners were asked to elaborate their understanding of the area of BI in general. Concerning this, two groups of answers can be identified. The first group is more technology-oriented, whereas the second group is more concept-oriented. For the first group of technology-oriented definitions, the IPs described BI as flexible storage, retrieval and querying of business-relevant data where large amounts of data can be aggregated in a meaningful way, or as IP3 puts it:

"Business Intelligence is an area, where I try to process data in a way to retrieve it again for statistical analysis, for data mining, with high-performance and highly aggregated. If I have to put it differently, I always say, yes, we are a huge graveyard of data which processes things in a way that users are able to quickly retrieve important data." (IP3)

Three of the six IPs put forward a more concept-oriented view concerning BI, where they describe it as a transformation of data into information, knowledge, and decisions. IP2 explains BI's ultimate goal as gaining knowledge and make correct decisions:

"[...] being able to gain insight into the company [...] make correct decisions or decisions that are as correct as possible under difficult circumstances, in a short amount of time, and facing continuous change also of the environment." (IP2)
As a main application area for BI, controlling and particularly the area of financial reports was pointed out most often. Apart from this, budgeting, quality management, contract management, personnel planning, marketing, sales, and customer relationship management (CRM) were mentioned. In line with controlling and financial reporting as main application area, the BI method mentioned most often, was reporting. Moreover, data warehousing, data modeling, analysis, data mining, OLAP (on-line analytical processing), ROLAP (relational on-line analytical processing), dashboarding, scorecarding, and decision support were mentioned as BI methods used by the interview partners. From a software infrastructure point of view, the product Cognos was stated most often followed by SAP. Other products that were mentioned are Business Objects, Hyperion, Microsoft, Marketing Manager, Micro Strategy, Oracle, and Siebel Analytics.

1) Advantages \& Critical Areas: The majority of perceived advantages of BI are related to the technologyoriented understanding of BI mentioned above:

- common data basis (across the company),

- ability to deal with large amounts of data,

- quick queries and comparison of alternatives,

- possibility of customized views for users,

- common layout and wording in reports across the company, and

- basis for fact-based decision making.

With regard to data modeling and querying, the idea of ad-hoc querying in contrast to pre-defined reports was emphasized. The main problem areas observed by the interview partners are data, interoperability, performance, and under-utilization. Regarding the first problem area of data, the interview partners mentioned three specific types. First, the problem of data timeliness - IP5 mentions the problem that data is entered into the system much too late due to organizational barriers and therefore, reports generated by the BI system are worthless, because they refer to data that is not up-to-date:

"[...][T]he main reason was that up to now, these (products) were entered into the system with a delay. [...] Sales had a contract that provided the profit-margin needed for this month, and this contract could not be seen. For him, everything was red." (IP5)

Second, some of the data needed for certain user groups is simply not available at all and third, the quality of the data itself is often very low. Another problem area identified by multiple interview partners is a technical obstacle: the lack of interoperability between different applications. Multiple software applications are often in use within one company and, due to the lack of interoperability, it is often cumbersome for users to carry out a certain task that might involve two or three different systems, which are not interconnected properly. Moreover, IP4 describes a problem related to users of BI systems: The power of the available tools is often 
underutilized which means that BI tools often have features and functionalities that are not used at all.

\section{B. Visualization}

In general, the use of visual methods in BI is very limited in the corporations of the interview partners. Only one IP reports regular usage of visual methods. Two IPs report limited application, one reports that only one visualization is in use, and in the companies of the two remaining IPs, no visualization is in use at all. Three IPs report that a possible reason for not using visualization might be found in corporate culture, personal taste, as well as trust.

"And this is consistent with my experiences, that decision-makers in (type of company) are extremely focused on numbers. If there really is a decision to be made, the numbers behind every chart want to be seen, however beautiful and dynamic the chart would be to use." (IP6)

Another possible reason identified by the IPs is that data problems are often more severe as mentioned in the previous section. And without the necessary data basis that is correct and complete, visualization does not make sense.

"The biggest problem of users and information consumers is not the way how these data are translated into information and how they are represented, but whether the data is correct or not." (IP2)

As reported by the IPs, the currently most prevalent form of data representation is a table format and visual methods are only at the beginning.

"Extremely often I see the generation of lists, which means the way how this could be represented differently is obviously only in its infancy." (IP2)

1) Advantages \& Critical Areas: When asked for the advantages of visual methods, the interview partners identified six main areas:

- understanding large amounts of complex data,

- easier for making comparisons,

- see relationships,

- see dynamic changes and trends,

- save time, and

- make daily work more attractive.

The first four advantages are more content-oriented, whereas the last two refer to organizational advantages. In the context of understanding large amounts of data, the power of visualization was mainly seen in gaining a quick overview and furthermore, making better decisions.

"For me, the basic advantage of visual methods is that

I can quickly gain an overview of the numbers." (IP5)

Regarding work attractiveness, IP5 states:

"That is surely nice to work with and makes everyday work more colorful and friendly. Also a cultural effect for work in a way that I do not always have to stick to the numbers." (IP5)
About the potential of dangers and disadvantages connected to visualizations, the IPs mentioned foremost

- possible information distortion,

- manipulation potential, and

- importance of choosing the appropriate technique.

With regard to the issue of manipulation potential and possible information distortion, IP5 stated:

"The disadvantages would be that I can disguise things easily. By using the usual tricks, via shortening, via logarithmic representations [...]” (IP5)

Apart from choosing the appropriate visualization technique, the critical area of choosing the right data was also mentioned (IP3). Interestingly, IP2 points out a possible downside of the advantage of making work more attractive as mentioned in the previous section. Using visual methods might also have negative effects in form of distraction.

"[...] the danger of drifting off into gaming. [...] Yes, because of just concentrating on visualization and presentation and using ways of presentation, one is distracted from the central issue which is analysis [...]" (IP2)

2) Tasks \& Techniques: Visualization techniques that were mentioned explicitly are bar charts, cockpits, highlighting, pie charts, and traffic lights. Only one IP mentions the use of maps for data representation. Star graphs were mentioned as the only multivariate visualization technique. IP4 states that the choice for the technique might also be determined by the personal taste of the users. From a task point of view, visual methods are mainly used for presentation, while analysis and exploration where cited as side issues.

"Because actually, from my point of view [...], these possibilities of visualization [...] are mostly used for general distribution, for communication, but not for gaining insights." (IP2)

In the sense of visual support for different business tasks, application areas were identified in operational, tactical, as well as strategic tasks. From a software point of view, Microsoft Excel has been mentioned by all interview partners as being used by users in their companies for different data analysis and also visualization tasks.

3) Users \& Application Areas: When asked about user groups that use or would benefit from visual methods in $\mathrm{BI}$, senior managers and controllers were mentioned most often. According to the majority of the interview partners, visual methods are received positively by users or are believed that they would be if introduced. On the one hand, according to the interview partners, users do not actively ask for visual methods but mainly think in terms of tables. On the other hand, IP1 reports that even though BI solutions are capable of generating diagrams no one uses them.

"Usually they come to me and say 'I need a table'. [...] If I visualize the table and show them what is possible, they are mostly very enthusiastic [...]" (IP3) 
An area identified for being particularly suitable for visualization techniques is to present continuously changing data. Moreover, customer relationship management, resource planning, and warehouse management were identified as potential application areas for visual methods.

\section{Interactivity}

When asked to describe what interactivity is, most interview partners focus on the areas of getting details on demand as well as so-called drill-down, a prominent concept in BI that describes the ability to go down to details of aggregated values. A very formal account on interactivity was given by IP4:

"Well, the spontaneous interaction with a diagram or the presented information at the time of observation and immediate feedback via an update." (IP4)

Interactivity is also seen as mediation of user-to-user communication (groupware), as interoperability between applications, and also as the possibility for data input by users. This illustrates that there is no common understanding of the concept of interactivity among IPs. Similar to visualizations in general, the usage of interactivity in particular is very limited. Most visual methods are reported to be static and interactivity itself as well as analysis is mostly secondary. When considering interactivity in tabular representation, IP1 reports that user defined data cubes are used only by very few users, but they use it extensively.

1) Advantages \& Critical Areas: The advantages of interactivity mentioned most often are flexibility and its potential to save time. In terms of flexibility, IPs mentioned that interactivity allows to retrieve exactly the information the user wants, and at the same time, the necessary amount of pre-defined reports decrease.

"This means, I go back and do not try to anticipate the information requirement, [...] that is most probably not correct anymore tomorrow because of the dynamics of the company and its environment but I delegate the decision 'What information do you need?' to the user, who is able to get the information that he needs via interactivity.” (IP2)

The potential of interactivity as a time saver is explained by the fact that no one else has to be asked and no specialized reports have to be developed. This implies that decisions can be made more quickly. Additionally, the help of interactivity in

- dealing with complexity,

- comparing alternatives and scenarios, as well as

- its positive effect for a deeper understanding with the result of making better informed decisions

were mentioned as advantages. Apart from these contentrelated advantages, IP5 and IP6 identified positive effects on work and creativity as similarly stated in the section about visualization.
On the potentially negative effects of interactivity, the interview partners mentioned

- distraction,

- higher costs,

- the need of more resources,

- that interactivity is more difficult to control,

- security issues, and

- user acceptance.

Distraction was also mentioned in connection with visualization in general and is seen as the danger of getting lost in details when having interactive visual tools. The issue of higher costs is explained by a higher degree of implementation effort. This also relates to the problem of security, with the questions of who can see which details and what levels of interactivity are available for which user or group. Moreover, a potential problem of interactivity is that it is more difficult to control because of an increased freedom for users. Furthermore, a higher amount of system resources is needed when providing interactivity in visual methods, which has negative effects on system performance as a whole. Finally, user acceptance was mentioned as a potentially critical area of interactivity, which might also turn into resistance.

"And if it really is beneficial for the consumer from an information content and handling point of view, for the information consumer it means change which is bad and therefore there is resistance." (IP2)

2) Users \& Application Areas: On the one hand, most IPs reported that users would react positively to (increased) interactivity or would most probably react positively if they knew more about interactivity in visualizations. On the other hand, users are reported to not actively ask for interactivity. IP3 explains that users not familiar with interactive visualzations, are satisfied with what they currently have, but users who have already experienced interactivity, want more.

“[...] Well, all users who do not know interactivity, are happy. [...] All users that have already seen what is possible by using interactivity want more [...]." (IP3)

Furthermore, almost all IPs think that interactivity can be beneficial. From a usage point of view, interactivity is seen as applicable for the tasks of communication, data input, explaining and finding causes, exploring alternatives, and detecting outliers. The interaction techniques that were mentioned to support these tasks were most often drilldown and details-on-demand. Besides this, configuration or the ability to change values and parameters, navigation in large information spaces, and grouping and filtering have been mentioned. However, when asked about the future development regarding interactivity in Visual BI, most IPs stated that this is not an issue for them. Only one of the interview partners stated that his company will actually invest more to put such methods into use. 


\section{DISCUSSION}

BI's main purpose is seen as supporting complex decisionmaking processes via transformation of data into knowledge and decisions. Moreover, the issue of fact-based decision making has been identified as one of the main advantages. However, as IP6 mentions, many business decisions are still made by gut feeling, even if the data was there. Two possible reasons can be identified for this: First, data that is available might not be accessible in a way to support decision making. Second, the lack of more widespread usage of BI might be more deeply rooted in corporate culture or personal taste of the responsible managers. In the first case, interactive visual methods could be a way to improve the current situation. The issue of corporate culture is going to be discussed further below.

Currently, the main application field of BI in practice is reporting in the sense of static presentation of data in reports, either as printable documents or electronically. Visual exploration and analysis, in turn, are not very important in current practice. They are mainly performed on an individual level using MS Excel as front-end.

Interestingly, trust among users towards visualizations seems to be lower than trust towards numbers as reported by the interview partners. As a possible reason for this, IPs mention the manipulative potential of visual representations where data might be distorted, whether intentionally or unintentionally, while a number stands for itself. This might also be caused by not enough knowledge about how visualizations work and how good quality charts could be designed. This possibly causes the fear of being manipulated without realizing it.

Interactivity and visualizations are reported to be generally two steps ahead. Specifically, visualization of data is seen as a potentially beneficial area but not considered very important in practice. A possible reason reported by the IPs is that still much more fundamental issues need to be tackled in the context of BI at the moment. These more fundamental issues are identified as data-related and form the basis of all further processing.

\section{A. Interactivity}

Because of the fact that visualization in general is not used broadly in practice, interactivity in visual methods is even less an important topic today. However, the interview partners had many ideas about possible uses and benefits of interactivity and also believe that users would react positively if they knew more about the possibilities of interactivity. This corresponds to the observation of a study on interactivity [15] that was conducted in a different context. Two reasons for the positive effect of interactivity given in theory [17], [18], [19] are the reduction of cognitive load (reducing the gulfs of execution and evaluation [20]) and a higher expressiveness of the user interface language (richer possibilities for input and output). Both these reasons were also recognized as benefits of interactivity in the empirical study. The reduction of cognitive load is largely seen as helping to make decisions more quickly and as saving time. The higher expressiveness of the user interface language is described as helpful in dealing with complexity and gaining a deeper understanding of the data. The third reason for the positive effect of interactivity is seen in theoretical approaches in higher engagement (feeling of being in control / first-personness) [17]. This was also recognized in the empirical study and interview partners claimed that increased use of interactivity and visual methods would create a more interesting and attractive working environment for employees. Furthermore, it was stated that this would enhance the creativity of employees tremendously and reflects research [21], [22]. This notion of creativity and interactivity is also related to the concept of being in the flow [23]. The term "flow" denotes an engaging experience and a level of immersion that is achieved by the user when experiencing a system. Arriving at this experience can be eased by providing means for seamless interactivity with tools and artifacts.

\section{B. Corporate Culture}

Apart from not knowing what is possible in terms of visual methods, users tend to stick to work habits they have employed and are largely defined by a certain corporate culture. In the area of usability engineering, this issue has been acknowledged [24]. A lesson learned from this might be that corporate culture is a crucial factor that needs to be considered when developing visual methods.

\section{Validity}

This empirical study can only be a first step towards exploring and understanding the multi-faceted phenomenon of interactivity in Visual BI. Its purpose is to shed some light on the users' perspectives and their understanding of visualization and interactivity. In lieu of in-depth empirical as well as theoretical work that concentrates on this issue, a qualitative approach was chosen to obtain a broader understanding of the topic from a user's point of view. However, due to the fact of the limited amount of participants, the acquired and presented results should be taken with caution. The results reflect the opinions and conceptualizations of six individuals who are experts in the field of Business Intelligence. Therefore, they cannot be generalized without reservation. However, no major contradictions where encountered among the interview partners which suggests at least some potential for broader validity of the obtained results. Moreover, the selected group of interview partners cannot be characterized as the classical end-users. Therefore, the opinions of the interview partners may differ from those of end-users without IT background and have to be put into perspective. 


\section{CONClusion \& Future Work}

In order to better understand interactivity and visual methods in the context of BI, an empirical study was carried out. Six qualitative interviews with IT experts of large Austrian companies have been conducted and analyzed. In the companies of the interview partners, the use of visual methods in general is very low in the field of BI and the use of interactivity in visual methods is even lower. However, a number of interesting insights were gathered in the study. Three of these aspects are corporate culture, work attractiveness, and creativity. Corporate culture appears to have a big influence on work practices and determines largely how work is done and problems are solved in a work environment. With regard to work attractiveness, interactive visual methods are believed to have a positive influence on daily work practice as reported by the interview partners. This is also connected to a special focus on the creativity of employees that can be used to achieve better business performance. All of these aspects describe a shift from a strict top down regime, where not only targets are preset but also the exact processes, to a more flexible approach that leaves more room for flexibility in employees. Moreover, the issue of trust seems to play an important role in the attitude towards visual methods. Trust in diagrams is reported to be lower than trust in numbers, and visual methods are often considered as nice to have as add-ons to make reports more flashy than as a tool for visual exploration or analysis. Furthermore, according to the interview partners, interactivity and visual methods are often not known to users and, therefore, no demand is created from the user side. Many benefits are identified in connection to interactivity, and most importantly for the business context, interactivity is associated with supporting a deeper understanding of data for making well-informed decisions by the interview partners. However, in current practice of BI, the role of interactivity in visual methods seems to be a secondary issue only and other more relevant problems, which are mainly data-related, need to be solved first.

\section{A. Hypotheses}

In Section III, a set of three hypotheses was formulated:

H1. Most visual methods currently applied in Business Intelligence are static or employ only very limited forms of interactivity.

This first hypothesis is supported by the findings of the empirical study. The use of visual methods is very limited in general and the majority of the used visualizations are static as reported by the interview partners.

$\mathrm{H} 2$. Increasing the interactivity of visual methods is desired by users.

The answer to this hypothesis is twofold. On the one hand, IT managers that were interviewed in the course of the study state that they would like to see more interactivity in visual methods themselves. On the other hand, it became apparent that the concept of interactivity in visual methods is not really known among end-users. This also means that interactivity is not actively asked for by end-users.

H3. According to users, interactivity aids information and knowledge gains in business data analysis.

The results of the empirical study show that the interviewed IT managers see interactivity as beneficial to gain information and knowledge. Similar to the previous hypothesis, end-users of the respective companies are reported to share this view but only if they have already experienced interactivity. Besides the perceived benefits, also critical aspects and disadvantages were identified.

\section{B. Future Work}

There is a clear need for empirical studies to investigate the role, advantages, and disadvantages of interactivity in visual artifacts in order to provide actionable evidence. Particularly, the question of trust depending on the form of representation needs to be investigated thoroughly to be able to answer the question why trust in diagrams is apparently lower than trust in numbers. Probably most important for stakeholders, the added value of both visual methods and interactivity needs to be demonstrated to users. This might be done via examples, empirical evidence, and, probably most successfully, through business success stories. Overall, Visual BI and the role of interactivity are extremely interesting and rich topics that will grow in importance in the future.

\section{ACKNOWLEDGMENT}

This work was supported by the Centre for Visual Analytics Science and Technology (CVAST; \#822746). Many thanks to Hanna Risku for feedback and support.

\section{REFERENCES}

[1] M. Grothe and P. Gentsch, Business Intelligence. Aus Informationen Wettbewerbsvorteile gewinnen. Addison-Wesley, München, 2000.

[2] S. Few, "BizViz: The Power of Visual Business Intelligence," http://www.b-eye-network.com/view/2470, Mar. 2007, accessed at: January 25, 2009.

[3] R. Spence, Information Visualization: Design for Interaction, 2nd ed. Upper Saddle River, NJ: Prentice Hall, 2007.

[4] P. Saraiya, C. North, V. Lam, and K. A. Duca, "An InsightBased Longitudinal Study of Visual Analytics," IEEE Transactions on Visualization and Computer Graphics, vol. 12, no. 6, pp. 1511-1522, 2006. 
[5] W. Aigner, "Understanding the Role and Value of Interaction: First Steps," in Proceedings of International Workshop on Visual Analytics (EuroVA 2011), S. Miksch and G. Santucci, Eds. Eurographics, May 2011, pp. 17-20.

[6] K. Sedig, P. Parsons, and A. Babanski, "Towards a Characterization of Interactivity in Visual Analytics," Journal of Multimedia Processing and Technologies, Special issue on Theory and Application of Visual Analytics, vol. 3, no. 1, pp. 12-28, 2012.

[7] H. Lam, E. Bertini, P. Isenberg, C. Plaisant, and S. Carpendale, "Empirical Studies in Information Visualization: Seven Scenarios," IEEE Transactions on Visualization and Computer Graphics, vol. 18, no. 9, pp. 1520-1536, 2012.

[8] P. Chamoni and P. Gluchowski, Analytische Informationssysteme: Business Intelligence-Technologien und-Anwendungen, 3rd ed. Springer, Berlin, 2006.

[9] T. H. Davenport and J. G. Harris, Competing on Analytics: The New Science of Winning. Boston: Harvard Business School Publishing, 2007.

[10] C. Howson, Successful Business Intelligence: Secrets to Making BI a Killer App. New York: McGraw-Hill Osborne Media, 2008.

[11] C. Bange, Werkzeuge für analytische Informationssysteme, 3rd ed. Berlin: Springer, 2006, pp. 89-110.

[12] T. Ahokas, "Information visualization in a business decision support system," Master's thesis, University of Helsinki, Faculty of Science, Department of Computer Science, 2008.

[13] J. Kohlhammer, T. Tekusova, and C. Bange, "Visual Business Intelligence," is report, vol. 12, no. 7+8, pp. 20-25, 2008.

[14] C. Bange, "Business Intelligence: Markt, Trends und Technologien," Presentation slides of Analytics Day at FraunhoferInstitut für Graphische Datenverarbeitung IGD Darmstadt, http://www.igd.fraunhofer.de/igd-a3/extern/va-day/2008/pdfs/ BI\%20Markt\%20Trends\%20Technologien\%2001-2008.pdf, Fraunhofer-Institut für Graphische Datenverarbeitung IGD Darmstadt, 2008.

[15] D. Richards, "Is Interactivity Actually Important?" in Proceedings of the 3rd Australasian conference on Interactive entertainment. Perth, Australia: Murdoch University, 2006, pp. 59-66.

[16] S. Carpendale, "Evaluating Information Visualizations," in Information Visualization: Human-Centered Issues and Perspectives (Lecture Notes in Computer Science). Berlin: Springer, 2008, pp. 19-45.

[17] E. L. Hutchins, J. D. Hollan, and D. A. Norman, "Direct Manipulation Interfaces," Human-Computer Interaction, vol. 1, no. 4, pp. 311-338, 1985.

[18] D. A. Norman, Things That Make Us Smart: Defending Human Attributes In The Age Of The Machine. Basic Books, 1993.
[19] S. K. Card, J. D. Mackinlay, and B. Shneiderman, Readings in Information Visualisation. Using Vision to Think.: Using Vision to Think. San Francisco: Morgan Kaufmann, 1999.

[20] D. A. Norman, Psychology of Everyday Things. Basic Books, 1988.

[21] B. Shneiderman, Leonardo's Laptop: Human Needs and the New Computing Technologies. Cambridge: The MIT Press, 2002.

[22] G. Fischer and E. Giaccardi, "Sustaining Social Creativity," Communications of the ACM, vol. 50, no. 12, pp. 28-29, 2007.

[23] M. Csikszentmihalyi, Flow: The Psychology of Optimal Experience. New York: Harper Perennial, 1991.

[24] D. A. Norman, "Design as Practiced," in Bringing Design to Software, T. Winograd, Ed. Michigan: ACM Press, 1996, pp. 233-247. 